\title{
À propos de la paréchèse chez Homère
}

\author{
Pierre-Yves Testenoire*
}

Homère est maître du style : en tant que source et modèle de cet art du dire qu'est la rhétorique, il n'est, pour les Anciens, pas un domaine qui touche à la parole où il ne soit exemplaire. Aussi est-il un modèle pour le travail de la matière sonore de la langue. On connaît les très belles pages que Denys d'Halicarnasse consacre à ce sujet ${ }^{1}$. Mais si dans les textes examinant les ressorts de l'euphonia homérique, les questions de rythme ou d'expressivité sonore sont richement traitées, l'analyse des phénomènes de répétitions phoniques dans l'Iliade et l'Odyssée reste peu développée. Les notions d'allitération ou d'assonance n'existent pas en tant que telles en grec ancien, ni d'ailleurs en latin où le terme n'apparaît qu'à la Renaissance. Le vers se conçoit, dans l'Antiquité, moins comme une chaîne dont il s'agit d'analyser la reproduction de séquences qu'un assemblage de mots ( $\left.\sigma \dot{v} v \theta \varepsilon \sigma 1 \varsigma \lambda \varepsilon^{\prime} \xi \varepsilon \omega v\right)$ plus ou moins harmonieux. Les phénomènes d'échos sonores, s'ils existent chez Homère, restent, en outre, relativement discrets. La poésie homérique n'est pas structurellement allitérative comme peuvent l'être les poésies archaïques dans d'autres langues. C'est pourquoi la notion de $\pi \alpha \rho \eta ́ \chi \eta \sigma ı \varsigma$ (paréchèse) qu'utilise l'Antiquité tardive paraît plutôt adaptée au corpus homérique et mérite ici toute notre attention.

\section{La paréchèse définie par Hermogène}

La notion de paréchèse apparaît assez tardivement dans l'histoire de la rhétorique

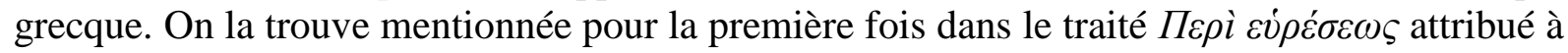
Hermogène. Elle apparaît dans le quatrième livre de ce traité où sont inventoriées les différentes figures de l'élocution. Après l'examen de l'antithèse, de la période, du pneuma, de la tension et du dilemme, un chapitre est consacré à la paréchèse :

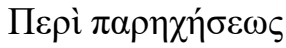

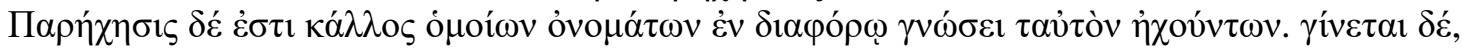

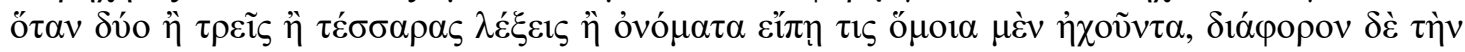

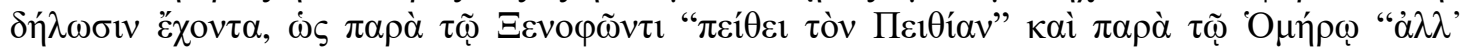



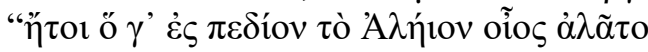

ôv $\theta 0 \mu o ̀ v \kappa \alpha \tau \varepsilon \delta \omega v, \pi \alpha ́ \tau o v ~ \alpha ̉ v \theta \rho \omega ́ \pi \omega v ~ \alpha ̉ \lambda \varepsilon \varepsilon i ́ v \omega v "$.

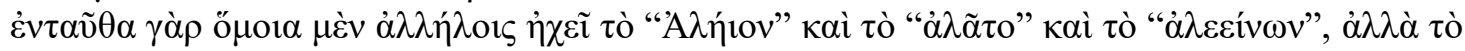

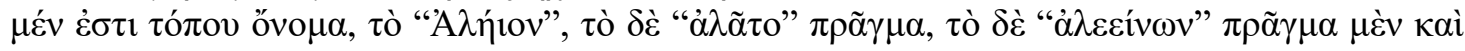

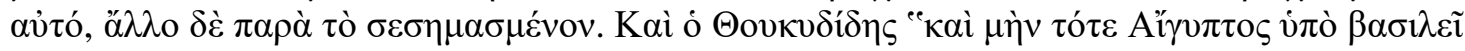





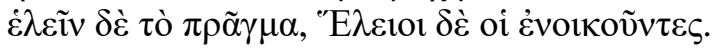

*Sorbonne Université, Laboratoire d'Histoire des Théories Linguistiques, UMR 7597.

1 Denys d'Halicarnasse, La Composition stylistique. Sur l'analyse d'Homère et l'euphonie propre aux vershomériques : VI, 3, 7-11; VI, 15, 11-16, 18 ; VI, 20, 8-22. 
La paréchèse est un bel effet produit par des mots semblables, au sens différent, qui répercutent le même son. Elle apparaît lorsque l'on utilise deux, trois ou quatre énoncés ou noms qui présentent des sons semblables tout en ayant une signification

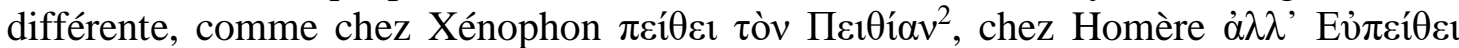
$\pi \varepsilon i ́ \theta$ ov $\mathrm{o}^{3}$ et, de manière très évidente, dans ce passage :

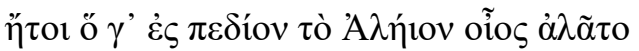

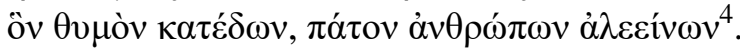

Ici, en effet, 'A $\lambda \hat{\eta} 10 v, \dot{\alpha} \lambda \tilde{\alpha} \tau o$ et $\dot{\alpha} \lambda \varepsilon \varepsilon i ́ v \omega v$ répercutent des sons semblables, 'A $\lambda \hat{\eta}$ est le nom d'un lieu, $\dot{\alpha} \lambda \tilde{\alpha} \tau$ o une action et $\dot{\alpha} \lambda \varepsilon \varepsilon i ́ v \omega v$ une action aussi, mais avec une autre

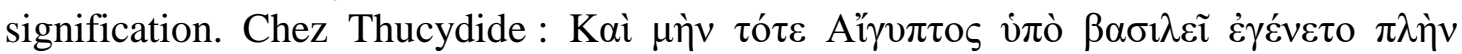



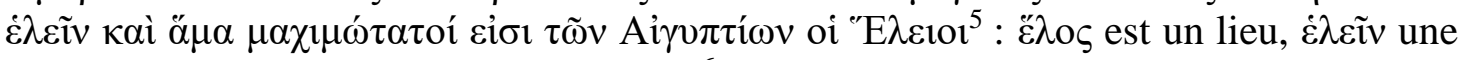
action et "E $\mathrm{\lambda} \varepsilon 10$ les habitants de la région".

La paréchèse est à classer parmi les figures d'expression jouant sur les sonorités. De ce point de vue, elle fait immanquablement songer aux figures gorgianiques que rapporte la

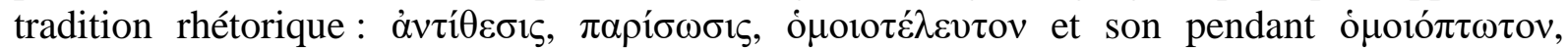
$\pi \alpha \rho v_{0} \mu \alpha \sigma i ́ \alpha . .$. Néanmoins la paréchèse n'est pas le rhabillage terminologique d'une de ces figures, et spécifiquement pas de celle dont elle semble le plus proche : la paronomase. En effet, si cette dernière repose également sur la similitude phonique entre deux mots - d'après les définitions et les exemples que l'on trouve dans la Rhétorique à Herennius ${ }^{7}$, chez Denys d'Halicarnasse $^{8}$, chez Quintilien ${ }^{9}$, Alexandre le rhéteur ${ }^{10}$ ou même ailleurs dans le corpus hermogénien ${ }^{11}$ - elle implique soit, par un procédé de dérivation, des mots d'une même famille soit, tout du moins, un jeu sur le signifié. La paronomase a souvent une vocation étymologique ou éponymique alors que, pour la paréchèse, la dimension sémantique est indifférente. Celle-ci apparaît donc bien comme une innovation du traité hermogénien Sur l'invention dont le quatrième livre en compte par ailleurs, d'après Michel Patillon, quelques autres ${ }^{12}$. La paréchèse est, comme son nom l'indique, un phénomène d'échos entre plusieurs lexèmes au sein d'un même énoncé.

Toute la difficulté, pour cette notion, est de déterminer la nature de la similitude entre deux mots qui se font écho. La paréchèse soulève la problématique inhérente à tous les phénomènes de répétitions sonores : celle de la frontière entre similitude et identité phoniques.

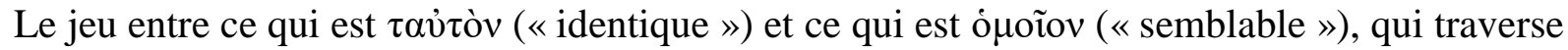
la définition, en témoigne. Deux mots formant paréchèse ne sont semblables que dans la mesure où ils contiennent certains éléments phoniques identiques. L'auteur du traité ne précise pas la part et la nature des éléments identiques nécessaires pour former une paréchèse. Dans chacun des quatre exemples donnés - deux pris dans la prose classique, chez Xénophon et Thucydide ;

2 Xénophon, Helléniques, VII, 1, 41.

3 Od. XXIV, 465-466.

${ }^{4}$ Il. VI, 201-202.

5 Thucydide, Histoire, I, 110, 2.

${ }^{6}$ Hermogène, Inv. [= $\pi \varepsilon \rho \grave{~ \varepsilon u ́ \rho \varepsilon ́ \sigma \varepsilon \omega \varsigma] ~ 4, ~ 7, ~ O p e r a, ~ p . ~ 194 . ~ S a u f ~ i n d i c a t i o n ~ c o n t r a i r e, ~ t o u t e s ~ l e s ~ t r a d u c t i o n s ~}$ contenues dans cet article sont nôtres.

${ }^{7}$ Rhétorique à Herennius, 4, 29.

${ }^{8}$ Thucydide, 48, 41 (3).

${ }^{9}$ Institution Oratoire, IX, 66-67.

${ }^{10}$ De Figuris, 36, 13-26.

${ }^{11}$ Sur les catégories du discours, 2, 5, 79-97.

${ }^{12}$ Cf. Hermogène, L'Art rhétorique, introduction par Michel Patillon, p. 98 sq. 
deux choisis dans les poèmes homériques - c'est toutefois la répétition d'une même syllabe qui fonde la paréchèse.

La figure, ainsi définie, est reprise par les productions scolaires de l'Antiquité tardive. Les technai rhétoriques et les nombreux commentaires aux traités attribués à Hermogène reprennent inlassablement les deux mêmes exemples homériques. La paréchèse se retrouve aussi chez certains scholiastes. Plusieurs paréchèses sont ainsi relevées dans les scholies aux Tragiques et, surtout, à Aristophane ; les scholies à Homère, en revanche, l'ignorent. Comme le corpus hermogénien connaît une fortune considérable dans l'Orient byzantin où il devient une œuvre de référence, un commentateur va faire un usage exponentiel de cette figure : Eustathe de Thessalonique. Plus d'une centaine de paréchèses différentes sont, en effet, relevées dans son immense commentaire de l'Iliade et de l'Odyssée. Surtout, il affine la théorie des paréchèses qu'on trouve exposée chez Hermogène. Par l'usage extensif qu'il fait de cette figure, par les développements théoriques qu'il y consacre, Eustathe s'attache à faire de cette figure tardive de la rhétorique grecque un outil d'analyse opérant pour les phénomènes sonores chez Homère.

\section{La « théorie des paréchèses » d'Eustathe}

Un développement, long et fort instructif, est consacré dans le commentaire d'Eustathe à cette notion de paréchèse. Ce développement théorique est introduit à l'occasion de la première paréchèse que le commentateur découvre au premier chant de l'Iliade :

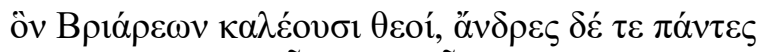

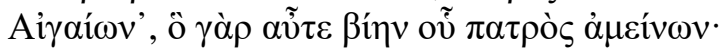

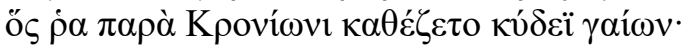

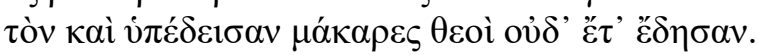

Monstre appelé par les dieux Briarée, et par tous les hommes

Egéon, qui surpasse même en violence son père,

Et qui s'assit auprès de Zeus dans l'orgueil de sa gloire !

Les Bienheureux, devant lui, prirent peur, renoncèrent aux chaines ${ }^{13}$.

Ces vers sont célèbres puisqu'ils contiennent le premier cas de double nomination qui figure dans les poèmes homériques ${ }^{14}$. C'est toutefois sur un autre aspect du passage que porte le commentaire d'Eustathe :

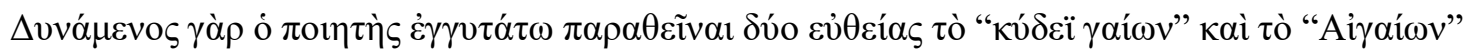



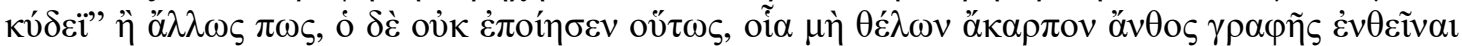

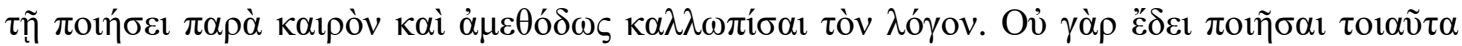

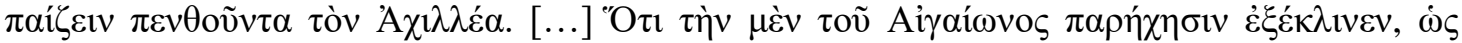

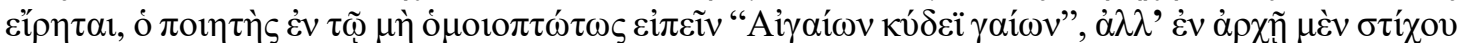



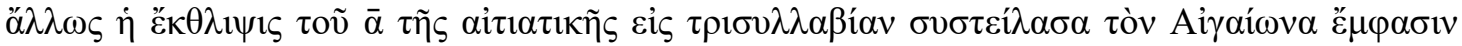

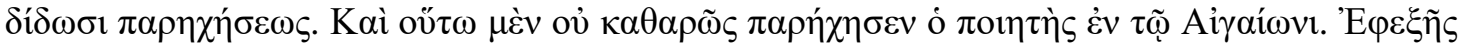



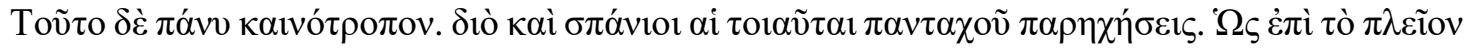

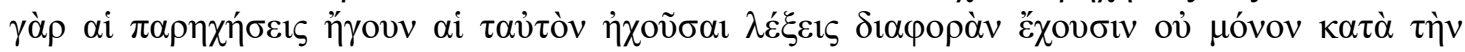

\footnotetext{
${ }^{13}$ Il. I, 403-406. Trad. de Ph. Brunet.

${ }^{14}$ Sur ces cas de double nomination et sur la « langue des dieux » qu'ils révèlent, on consultera notamment les travaux de Françoise Bader.
} 



oĩos à $\lambda \tilde{\alpha} \tau o \pi \alpha ́ \tau o v ~ \alpha ̉ v \theta \rho \omega ́ \pi \omega v ~ \alpha ̉ \lambda \varepsilon \varepsilon i ́ v \omega v "$


et ainsi faire une épanastrophe ou une paréchèse rhétorique plus audacieuse, comme

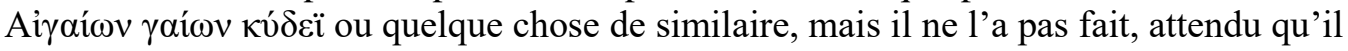
ne voulait pas insérer dans son poème un éclat de style stérile et embellir son discours mal à propos et sans méthode. Il ne convenait pas, en effet, qu'Achille fasse de telles plaisanteries alors qu'il était affligé. [...] Le poète a évité, comme il a été dit, la

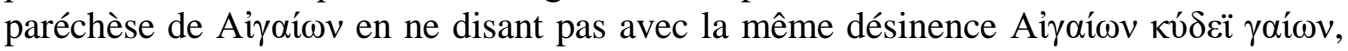
mais il a placé au début d'un vers Air $\alpha i ́ \omega v \alpha$ et à la fin d'un autre Airoí $\omega v$, c'est-à-dire un accusatif et un nominatif, et c'est d'ailleurs l'élision du $\alpha$ de l'accusatif, ayant réduit Air $\alpha i ́ \omega v \alpha$ à trois syllabes, qui donne l'apparence d'une paréchèse. Ainsi le poète n'a pas fait de paréchèse pure avec Aijoí $\omega v$. Tout de suite après, il emploie une autre paréchèse

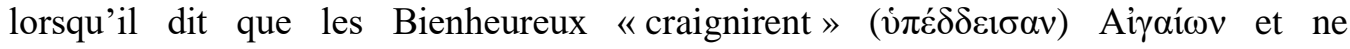
«l'enchaînèrent» pas ( $(\delta \delta \eta \sigma \alpha v)$. Ce phénomène est d'une espèce vraiment nouvelle. Aussi de telles paréchèses sont-elles extrêmement rares. Car, en outre, les paréchèses, c'est-à-dire les mots qui répercutent le même son, sont différentes non seulement du point de vue de la signification mais aussi de la prononciation comme Ev̉ $\pi \varepsilon \dot{\theta} \theta \varepsilon 1$

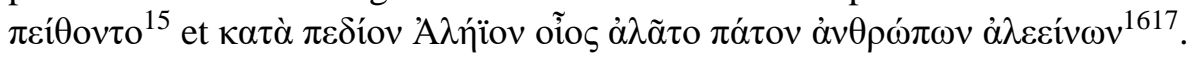

La richesse du passage, qui réunit en l'espace de trois vers deux paréchèses, justifie le développement théorique qui suivra. La première paréchèse est discrète, du fait de l'espacement des termes qui se répondent. La seconde, bien plus voyante, réunit dans un seul vers deux verbes ( et le êta se prononcent, en effet, tous les deux $i$ au XII ${ }^{\mathrm{e}}$ siècle du fait des progrès du iotacisme. Le commentaire cite ensuite le texte d'Hermogène, en reprenant, à quelques détails près, sa définition et ses deux exemples homériques.

C'est à présent qu'Eustathe innove par rapport à la définition devenue canonique d'Hermogène. Il affine la catégorie de la paréchèse en y introduisant des subdivisions :

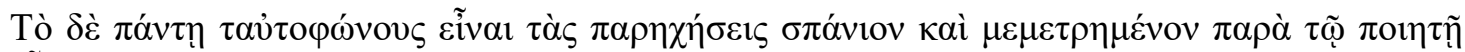

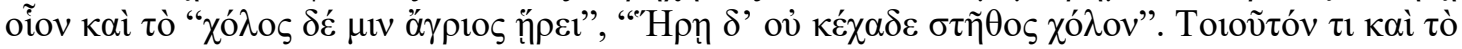

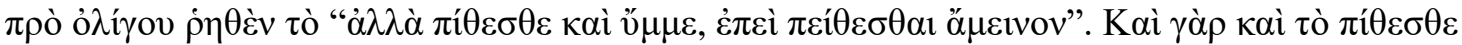

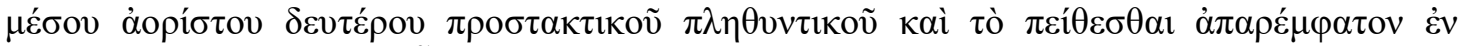

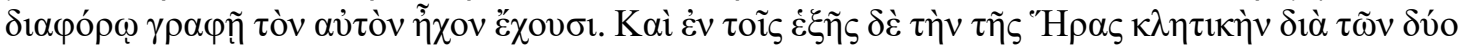



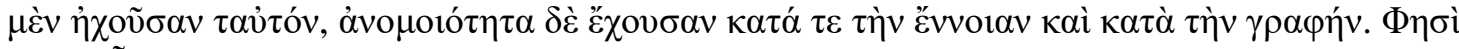



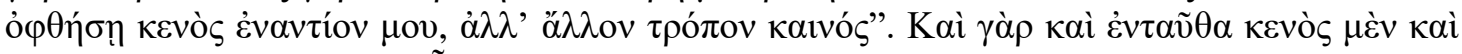

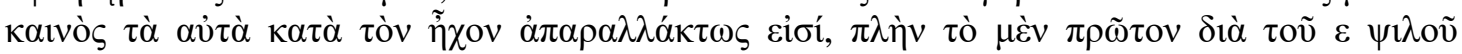

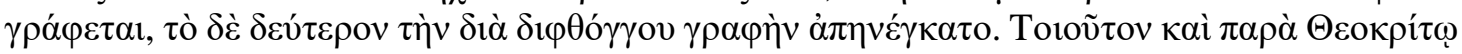

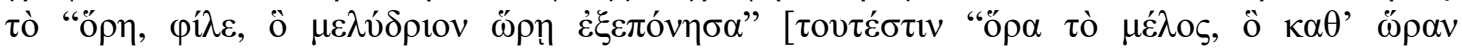

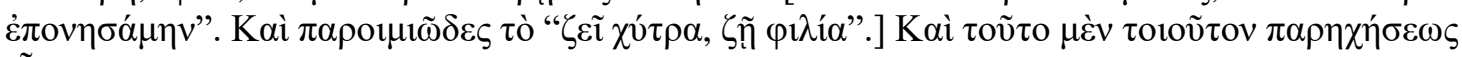

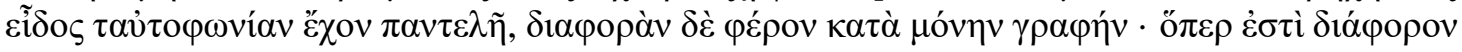

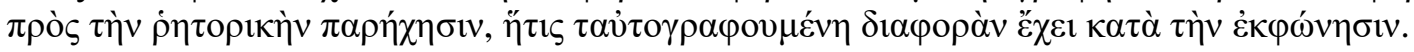

Il est rare et limité chez le poète que les paréchèses aient en tout point le même son



${ }^{15}$ Od. XXIV, 465-466.

${ }^{16} \mathrm{Il}$. VI, 201-202.

${ }^{17}$ Eustathe, Commentarii ad Homeri Iliadem, éd. par Van der Valk, I, 191.19-192.33.

${ }^{18}$ Il. VIII, 460-461. 


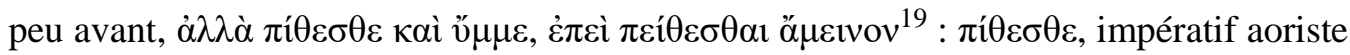
moyen à la deuxième personne du pluriel, et $\pi \varepsilon i ́ \theta \varepsilon \sigma \theta \alpha 1$, infinitif, présentent, avec une lettre différente, le même son. Plus loin, le poète place le vocatif de "H $\eta$ et celui d'"I $I$ ı s avec ses deux 1 proches l'un de l'autre conformément à ce genre de paréchèses qui répercute parfaitement le même son, mais qui présente des différences au

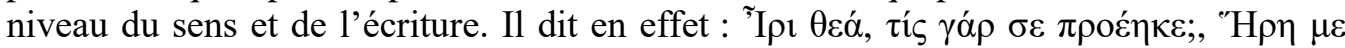

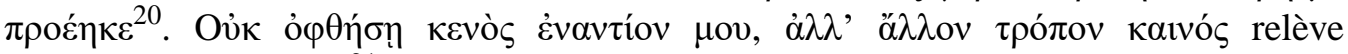


ce qui concerne le son, sauf que le premier est écrit avec un $\varepsilon$ pur et que le second est formé au moyen d'une diphtongue. Une figure du même genre se trouve chez Théocrite :

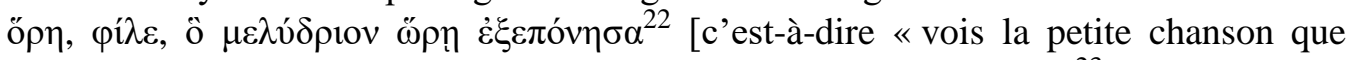

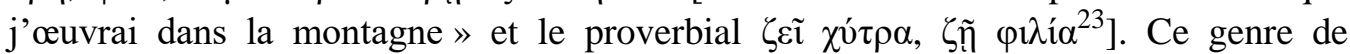
paréchèse repose sur une parfaite identité de prononciation, mais comporte une différence seulement au niveau de l'écriture. Ce qui est différent de la paréchèse rhétorique qui, supposant une identité d'écriture, comporte une différence au niveau de la prononciation ${ }^{24}$.

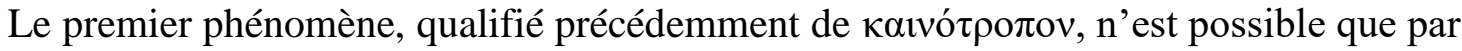
l'effet du iotacisme et la perte de la distinction phonémique des voyelles longues et brèves. Il est très bien représenté dans le commentaire d'Eustathe. À ce type de paréchèses supposant une identité phonique malgré une dissemblance graphique répond, dans la suite du texte, le phénomène inverse : une paréchèse reposant sur une identité graphique et une dissemblance

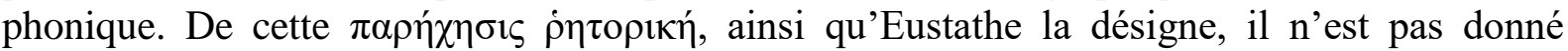
d'exemples, ceux repris un peu plus haut au corpus hermogénien relevant, selon toute évidence, de ce second type. Enfin, pour compléter la symétrie constituée par ces deux formes de paréchèse, s'ajoute une troisième catégorie :

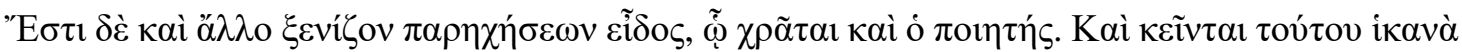

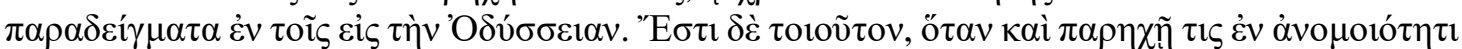



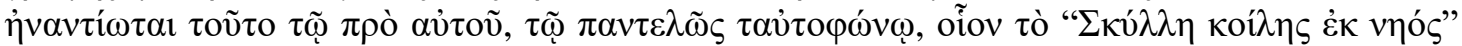

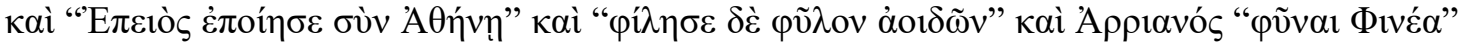

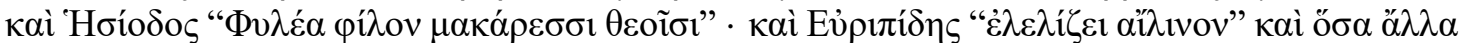

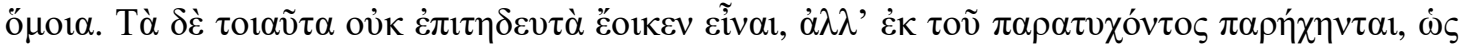

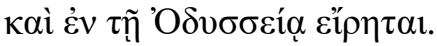

Et il y a un autre genre de paréchèse, d'aspect étrange, dont use le poète. Ses exemples se trouvent dans l'Odyssée. Il y a une telle figure lorsque l'on fait se répercuter des voyelles, avec une différence d'écriture, comme précédemment, mais que l'on altère quelque peu cette identité de prononciation. De telle sorte que ce cas se distingue du

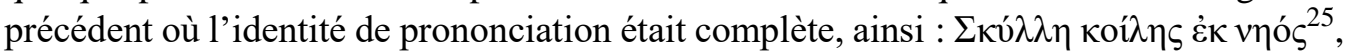

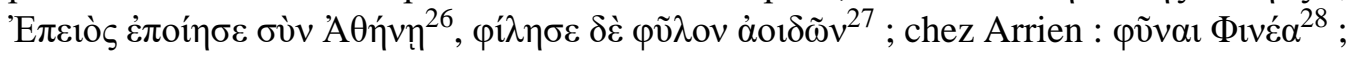

\footnotetext{
19 Il. I, 274.

20 Il. XVIII, 182-184.

21 «fort. Eust. Ipse» (Note de Van der Valk).

22 Théocrite, Idylle, VII, 51.

23 Zenob. IV, 12.

${ }^{24}$ Eustathe, Commentarii ad Homeri Iliadem, I, 192.33-193.18.

25 Od. XII, 245.

26 Od. VIII, 493.

27 Od. VIII, 481.

28 Arrien, Bithynicorum Frag. 20.
} 


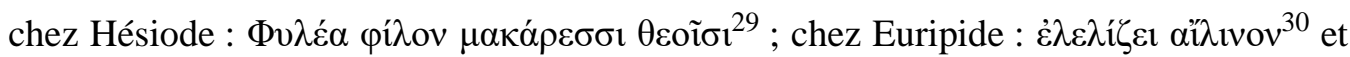
d'autres cas semblables. De telles expressions ne semblent pas être créées intentionnellement, mais bien se répercuter par hasard, comme on l'a dit dans l'Odyssée $e^{31}$.

Le troisième type, dégradé, de paréchèse correspond aux mots que le iotacisme a rendus non pas, comme dans le premier cas, parfaitement identiques, mais simplement similaires du point de vue phonétique. Les dissemblances qui subsistent, malgré l'uniformisation vocalique, tiennent aux cadres consonantiques différents. Cette troisième catégorie est abondamment illustrée. C'est l'abondance même des exemples qui semble d'ailleurs soulever le soupçon quant à l'intentionnalité du phénomène. Après l'examen de ce type de paréchèses, qualifié de

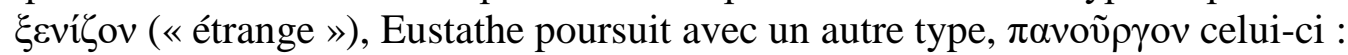

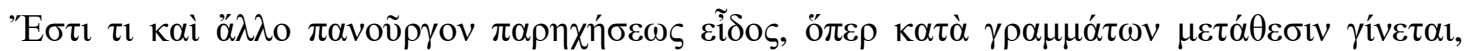

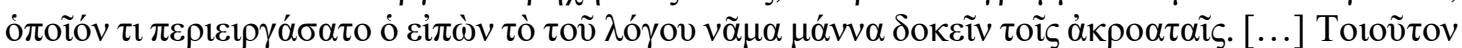

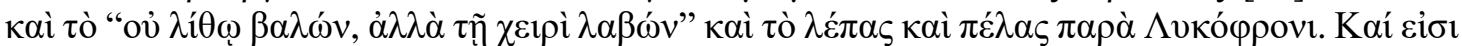

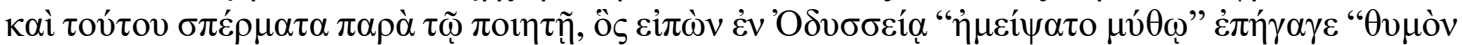

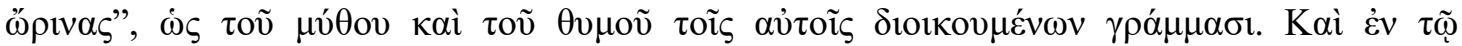

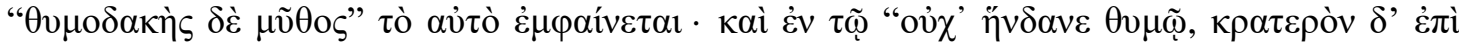
$\mu \tilde{v} \theta \mathrm{ov}$ हैं $\varepsilon \lambda \lambda \varepsilon v "$.

Il y a également un autre genre de paréchèse, qui nécessite de l'habileté, et qui survient par changement de lettres à peu près comme cet écrivain qui a déployé son habileté pour dire que le «ruisseau» $(\nu \tilde{\alpha} \mu \alpha)$ de son discours était une «manne» $(\mu \alpha \dot{v} v v \alpha)$ pour ses

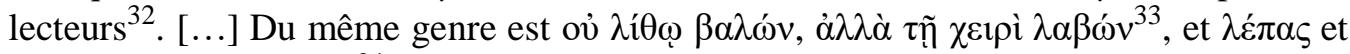
$\pi \dot{\varepsilon} \lambda \alpha \varsigma$ chez Lycophron ${ }^{34}$. Les germes de ce procédé sont chez le poète qui, lorsqu'il dit



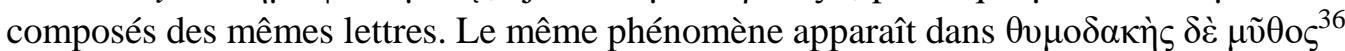

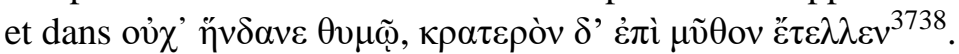

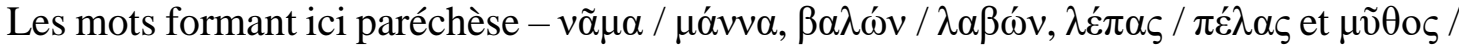
$\theta v \mu o ́ s$ - ne supposent pas, comme précédemment, l'itération d'une même syllabe, mais une inversion de consonnes. Ce phénomène est désigné sous un autre nom dans le reste du commentaire d'Eustathe, celui d'òv $\alpha \gamma \rho \alpha \mu \mu \alpha \tau \imath \sigma \mu o ́ c$. Aussi n'est-ce pas un hasard s'il est fait mention du poète Lycophron, auquel une tradition attribue l'invention de cette figure. Six cas d'anagrammes homériques sont repérés dans le commentaire d'Eustathe, dont trois tiennent à

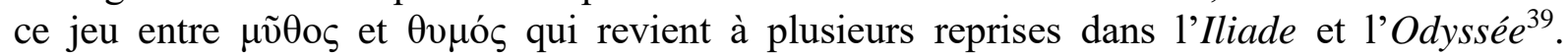
L'anagramme antique serait donc une sous-catégorie de la paréchèse : l'écho paréchétique entre deux énoncés s'obtiendrait soit par répétition soit par métathèse syllabique.

${ }^{29}$ Hésiode, frag. 176, 4 M. W. = fr. 93, 4 Rz.

${ }^{30}$ Euripide, Phéniciennes, 1514-1519.

${ }^{31}$ Eustathe, Commentarii ad Homeri Iliadem, I, 193.18-30.

32 «Christianus auctor mihi ignotus » (note de Van de Valk).

33 «Locus ignotus, ut opinor » (note de Van de Valk).

${ }^{34}$ Lycophron, Alexandra, 419-420.

35 Od. XV, 485 sq.

${ }^{36} \mathrm{Od}$. VIII, 185.

${ }^{37}$ Il. I, 378-379.

${ }^{38}$ Eustathe, Commentarii ad Homeri Iliadem, I, 193.30-37.

${ }^{39}$ Pour un aperçu plus complet de la question des anagrammes dans l'Antiquité grecque et des similitudes avec la paréchèse d'Eustathe, nous nous permettons de renvoyer à notre étude : «Des anagrammes chez Homère?». 
Si le domaine de la paréchèse semble s'étendre chez Eustathe, il ne s'agit néanmoins pas d'y inclure tous les jeux sur les sonorités rencontrés dans les poèmes. Aussi le commentateur s'attache-t-il à exclure certains procédés :



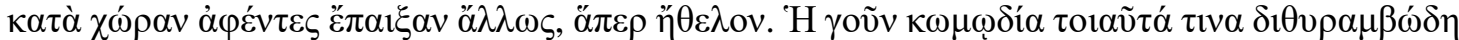

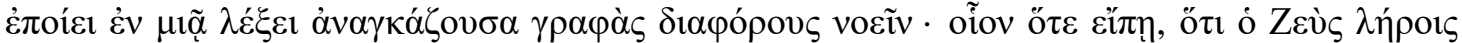

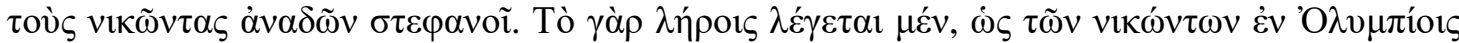



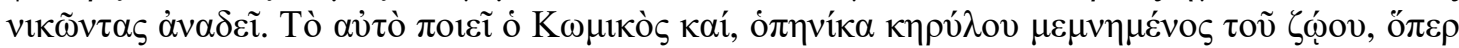

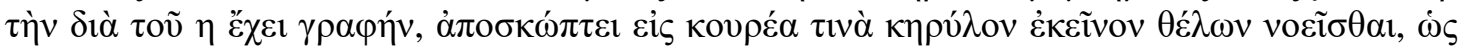

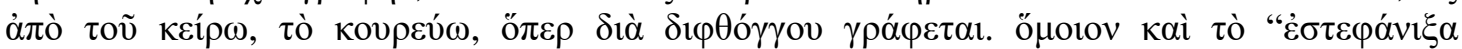





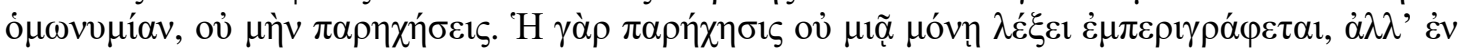

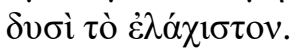

Après avoir ainsi travaillé un mot et l'avoir détourné, d'autres se sont amusés, d'une manière différente, à le laisser à la place qu'ils voulaient. La comédie, par exemple, créait des effets de dithyrambe avec un seul mot lorsqu'elle contraignait l'auditeur à avoir des écritures différentes à l'esprit, comme lorsqu'il est dit que Zeus couronne les vainqueurs de « radotages » $(\lambda \eta ́ \rho o 1 \varsigma)^{40}$. Il est question de « radotages » $(\lambda \eta ́ \rho o 1 \varsigma)$, car les vainqueurs à Olympie sont fascinés par les bavardages et l'emphase creuse, mais on veut dire qu'il couronne les vainqueurs de « lis » ( $\lambda$ sipíors) c'est-à-dire de fleurs. Le poète


s'écrit avec un $\eta$, il lance une pique à un barbier avec l'intention que cet «alcyon »

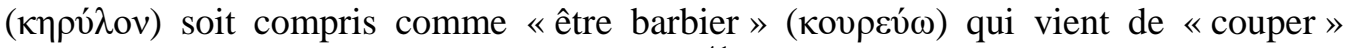

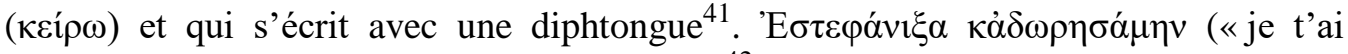

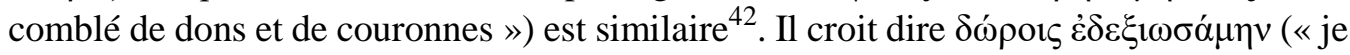
t'ai comblé de présents »), mais il fait des fautes et parle en dorique, car $\dot{\varepsilon} \sigma \tau \varepsilon \varphi \alpha ́ v 1 \xi \alpha$ relève du dialecte dorique. Ces plaisanteries comiques reposent, à vrai dire, sur des similitudes entre des noms, ce ne sont cependant pas des paréchèses. Car la paréchèse ne repose pas sur un seul mot, mais au moins sur deux ${ }^{43}$.

Est ainsi exclu de la paréchèse ce qu'on appelle communément le jeu de mots, c'est-àdire l'allusion à un mot absent de l'énoncé ayant une ressemblance phonétique avec celui effectivement employé. Les exemples sont ici empruntés à Aristophane ; mais de tels jeux existent, on le sait, chez Homère. Outre les calembours, les plaisanteries savantes et les jeux sur l'intertextualité, bref tout ce qui fait appel au bagage linguistique ou culturel du lecteur ou de l'auditeur, sont tenus pour étrangers à la paréchèse. La dernière phrase du paragraphe - «la paréchèse ne repose pas sur un seul mot, mais au moins sur deux »-marque, à cet égard, une frontière déterminante pour la délimitation de la figure. Les échos de la paréchèse se placent uniquement sur l'axe syntagmatique du vers ou de l'énoncé; tout ce qui relève du paradigmatique est forclos. Enfin, Eustathe récapitule les étapes du raisonnement parcourues, formant ainsi la $\pi \alpha \rho \eta \chi \eta ́ \sigma \varepsilon \omega \nu ~ \theta \varepsilon \omega \rho i ́ \alpha$ :



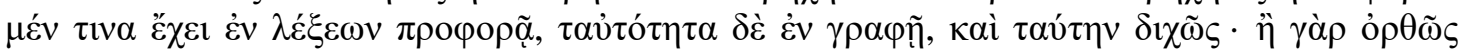

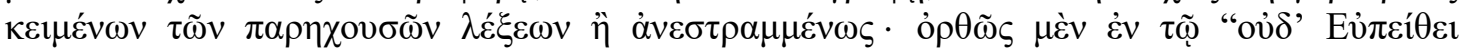



\footnotetext{
${ }^{40}$ Aristophane, Ploutos, 587 sq.

41 Aristophane, Oiseaux, 299-300.

42 Aristophane, Cavaliers, 1225.

43 Eustathe, Commentarii ad Homeri Iliadem, I, 193.37-194.13.
} 




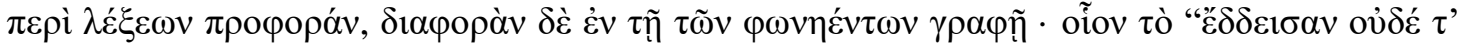

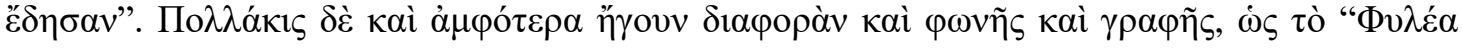

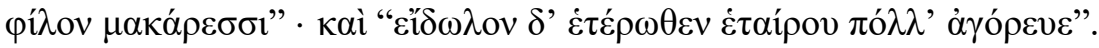

Telle est donc, en résumé, la théorie des dites paréchèses. Soit la paréchèse présente une différence dans la prononciation des mots et une identité dans l'écriture, et cela de deux façons : soit les mots qui se font écho sont disposés dans le bon ordre, soit ils le sont dans un ordre bouleversé. Ils sont dans le bon ordre dans ov̉ ${ }^{\prime}$ Ev̉ $\pi \varepsilon i ́ \theta \varepsilon 1 \pi \varepsilon i ́ \theta o v \tau 0^{44}$ et dans les exemples du même type ; ce qui est, à vrai dire, le seul cas de paréchèse vraiment satisfaisant. Mais ils sont dans un ordre bouleversé dans les cas comme $\mu \tilde{v} \theta$ o $\varsigma$ et $\theta v \mu o ́ c$, $\beta \alpha \lambda \omega ́ v$ et $\lambda \alpha \beta \omega ́ v$, et ainsi de suite. Soit, au contraire, elle présente une identité au niveau de la prononciation des mots et une différence dans l'écriture des voyelles, comme :

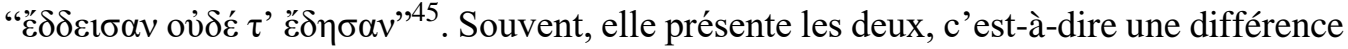

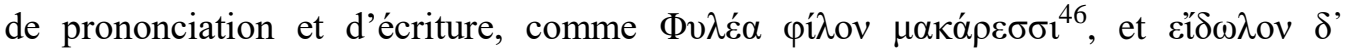

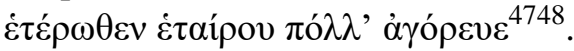

S'il est pris tant de soin à l'exposé des principes et des subdivisions de cette figure, c'est qu'il s'agit d'un outil d'analyse dont Eustathe use abondamment. Chacune des paréchèses découvertes au fil de son commentaire peut être ramenée à l'une des trois catégories ainsi hiérarchisées : la paréchèse due à un écho phonique originel, la paréchèse due à un écho phonique induit par des changements de prononciation, enfin, la forme dégradée de la paréchèse, celle qui malgré les changements de prononciation n'offre qu'un écho phonique imparfait. Ces catégories, définies ici par le recours à la diachronie, sont exprimées chez Eustathe en termes de correspondance entre la graphie et la phonie.

Après l'examen théorique de la paréchèse, il convient d'en étudier la mise en application dans la pratique du commentaire homérique. Le catalogue complet des paréchèses relevées par Eustathe excèderait les limites de cet article. Aussi, nous nous contenterons de proposer, à partir de quelques exemples, un panorama de la variété des figures présentes dans le commentaire d'Eustathe, illustrant l'originalité de la démarche de l'exégète dans le maniement de cet outil.

\section{La pratique de la paréchèse dans le commentaire d'Eustathe}

Les paréchèses qui relèvent de la première catégorie - la seule qui, selon Eustathe, soit vraiment satisfaisante - peuvent concerner des mots d'une grande banalité :



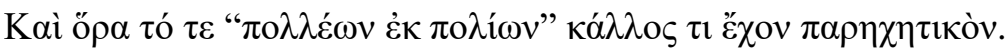

On voit que $\pi 0 \lambda \lambda \dot{\varepsilon} \omega v \dot{\varepsilon} \kappa \pi 0 \lambda i ́ \omega v$ forme un embellissement paréchétique ${ }^{49}$.

${ }^{44}$ Il. XXIV, 465-466.

${ }^{45}$ Il. I, 404-405.

${ }^{46}$ Hésiode, fr. 176, 4 M. W. = fr. 93, 4 Rz.

${ }^{47}$ Od. XI, 83.

${ }^{48}$ Eustathe, Commentarii ad Homeri Iliadem, I, 194.13-22.

${ }^{49}$ Eustathe, Commentarii ad Homeri Iliadem, I, 292.33. 
Ce type de paréchèse, souvent qualifié par l'adjectif $\sigma \omega ́ \varphi \rho \omega v$, porte indifféremment sur

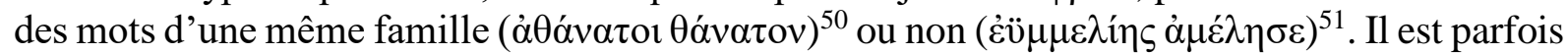
couplé avec d'autres figures, épanastrophes, parisoses ou, le plus souvent, avec une figure étymologique :

b) $I l$. VII, 5-6

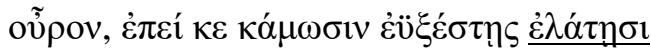

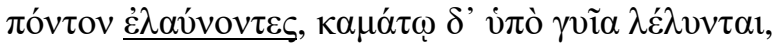

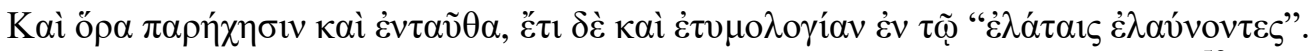

On voit ici une paréchèse, et une étymologie dans $\dot{\varepsilon} \lambda \alpha \dot{\alpha} \tau \alpha 1 \zeta \dot{\varepsilon} \lambda \alpha v ́ v o v \tau \varepsilon \varsigma^{52}$.

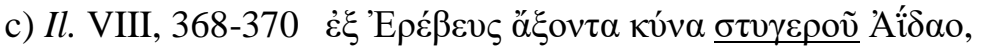

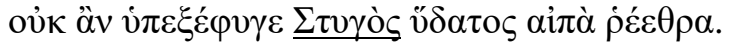

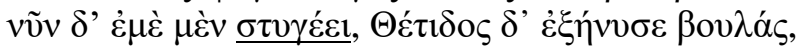

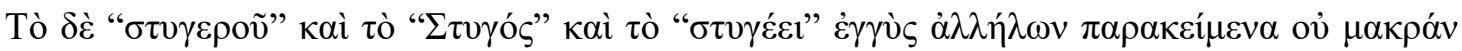

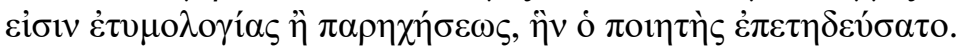

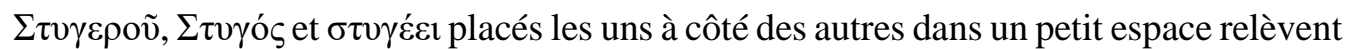
de l'étymologie ou de la paréchèse, que le poète a créée intentionnellement ${ }^{53}$.

Une question, récurrente dans le commentaire d'Eustathe au sujet de la paréchèse, concerne l'espacement convenable entre les deux termes qui se répondent. Trop loin, l'écho n'est plus perceptible, trop près, il relève de la plaisanterie de potache :



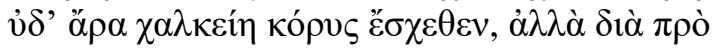

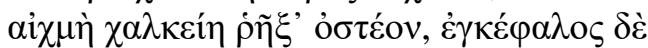

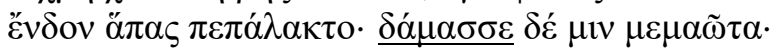

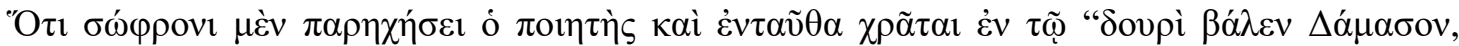

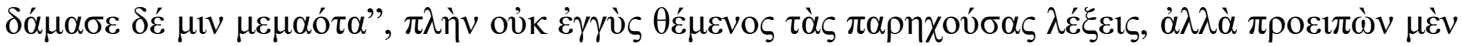

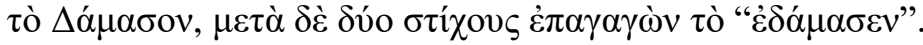

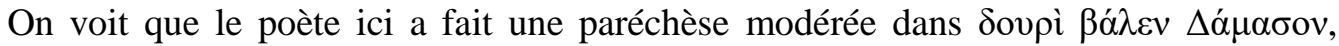

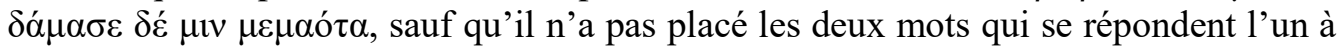

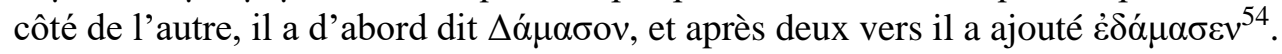

Si la paréchèse nécessite parfois un espacement décent, certains sujets sont aussi à éviter pour des raisons de bienséance. De même qu'Achille, étant donné son chagrin, ne pouvait se laisser aller à une paréchèse éclatante sur le nom Air $\alpha i \omega v^{55}$, de même Eustathe félicite Homère de ne pas faire, dans l'épisode d'Eumée, de paréchèse sur le nom du cochon ${ }^{56}$. Dans le même ordre d'idées, le commentateur sait gré au poète de toujours éviter, dans l'Iliade, les paréchèses


paréchèses qu'Homère a épargnées à ses lecteurs sont nombreux dans le commentaire d'Eustathe. En voici un cas :








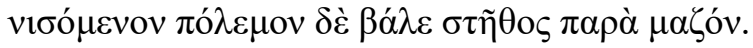

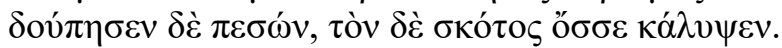



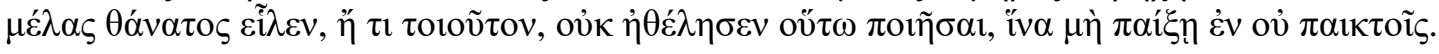

Ici on voit qu'Homère aurait pu faire une paréchèse à propos de Melanippe en disant

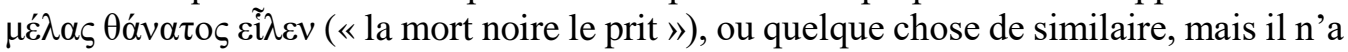
pas voulu la créer pour ne pas faire de plaisanterie dans les passages non amusants ${ }^{58}$.

Le deuxième type de paréchèse est également bien représenté dans le commentaire de l'Iliade et de l'Odyssée. L'identité phonique reconnue est le plus souvent due aux effets du iotacisme :

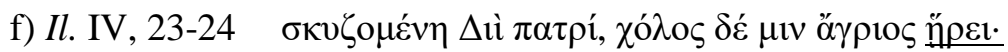

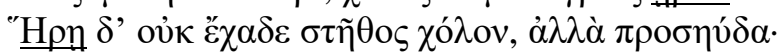

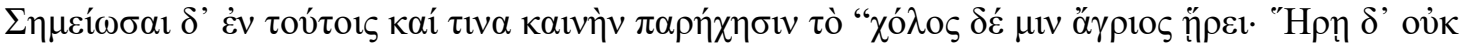

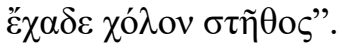

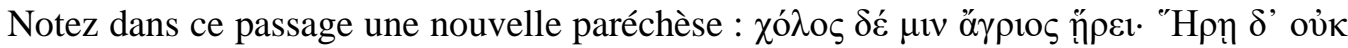

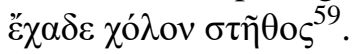

Plus rarement, l'identité est, comme ici, imputable à la perte de la distinction phonémique entre la voyelle longue et la voyelle brève :

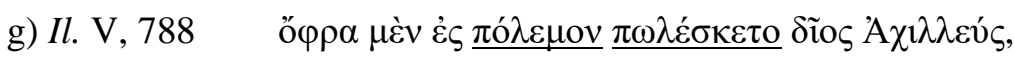



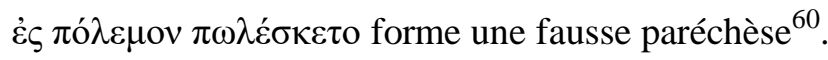

Pour qualifier les paréchèses de ce deuxième type, Eustathe reprend l'adjectif кaıvó $\varsigma$

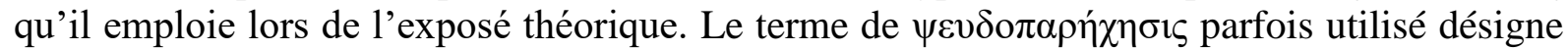
surtout les paréchèses de la troisième catégorie. Celles-ci sont, en principe, de qualité médiocre. Paradoxalement, ce ne sont pas les moins intéressantes pour l'exégèse. Ainsi la paréchèse notée ci-dessous, renforcée par la position des mots dans le vers - en début de vers et après la pause bucolique - rend compte d'un phénomène d'écho incontestable :

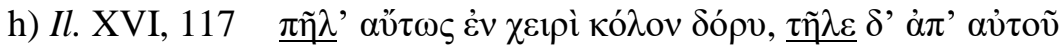

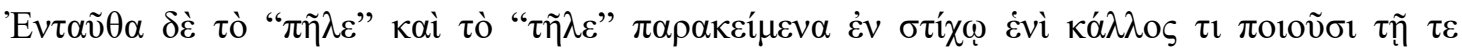

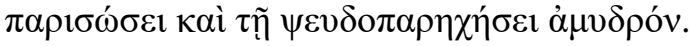

Ici $\pi \tilde{\eta} \lambda \varepsilon$ et $\tau \tilde{\eta} \lambda \varepsilon$ placés dans un seul vers créent, par la parisose et la fausse paréchèse, une beauté obscure ${ }^{61}$.

Enfin, il convient de citer un exemple des très nombreux cas de paréchèse involontaire qui forment l'essentiel de cette troisième catégorie :

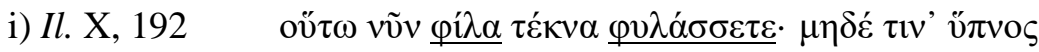

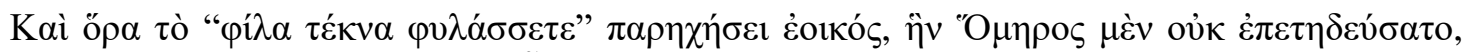

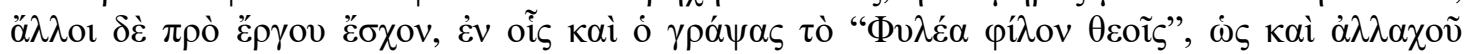
$\delta \eta \lambda \mathrm{v} \tau \alpha \iota 1 \pi \lambda \alpha \tau \dot{\tau} \tau \varepsilon \rho \circ$.

\footnotetext{
${ }^{58}$ Idem, III, 767, $17 \mathrm{sq}$.

${ }^{59}$ Idem, I, 695, 23 sq.

${ }^{60}$ Idem, II, 202, 15 sq.

${ }^{61}$ Idem, III, 818, 3 sq.
} 


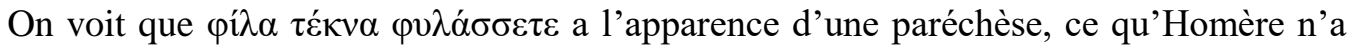
pas créé intentionnellement, mais que d'autres ont repris pour leur œuvre, parmi lesquels

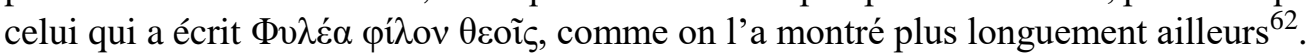

En définitive, l'approche des paréchèses vaut sans doute moins par sa spécificité que par sa quantité. Un certain nombre des paréchèses relevées par Eustathe sont, en effet, consignées ailleurs - dans des traités, des commentaires, des scholies - avec une autre terminologie. Il faut signaler, en outre, qu'à l'intérieur même du commentaire d'Eustathe un phénomène ici noté comme relevant de la paréchèse sera noté ailleurs comme relevant de la parétymologie ou de la paronomase. Des phénomènes d'échos inouïs dans les poèmes homériques sont néanmoins mis en lumière par le maniement récurrent de cette figure. La théorie des paréchèses atteste l'existence, à l'époque byzantine, d'une tradition d'exégèse homérique particulièrement attentive et réceptive aux jeux de sonorités. Cette tradition, en ce qui concerne les paréchèses, n'est pas celle des scholies. Le commentaire d'Eustathe en est un des meilleurs témoins.

\section{Bibliographie}

\section{Sources}

Allen, Thomas W. et Monro D. B. (éd.), Homeri Opera, Oxford, Oxford University Press, 1902-1908.

Brunet, Philippe (trad.), Homère, Iliade, Paris, Seuil, 2010.

Dindorf G. (éd.), Scholia graeca in Homeri Odysseam, Oxford, Clarendon Press, 1855.

Erbse, Hartmut (éd.), Scholia graeca in Homeri Iliadem, Berlin, De Gruyter, 1969-1988.

Jaccottet, Philippe (trad.), Homère, Odyssée, Paris, La Découverte, 1982.

Patillon, Michel (trad., comm.), Hermogène, L'Art rhétorique : exercices préparatoires, états de cause, invention, catégories stylistiques, méthode de l'habileté, Lausanne, Paris, L'Âge d'Homme, 1997.

Rabe, Hugo (éd.), Hermogène, Opera, Leipzig, Teubner, 1913.

Van der Valk, Marchinus (éd.), Eustathe de Thessalonique, Commentarii ad Homeri Iliadem pertinentes, Leyde, Brill, 1976-1987.

Weigel, J. A. G. (éd.), Eustathe de Thessalonique, Commentarii ad Homeri Odysseam, Hildesheim, G. Olms, 1970 (1825-1826).

\section{Études}

Bader, Françoise, La langue des dieux ou l'hermétisme des poètes indo-européens, Pise, Giardini, 1989.

Bader, Françoise, Anagrammes et allitérations, Paris-Louvain, Peeters, 1993.

Bérard, Victor, Introduction à l'Odyssée, vol. I, Paris, Les Belles Lettres, 1924.

Bernhardt, Gualterus, De alliterationis apud Homerum usu, Leipzig, Gotha, 1906.

Desbordes, Françoise, La Rhétorique antique, Paris, Hachette, 1996.

${ }^{62}$ Idem, III, 43, 17 sq. 
Dickey, Eleanor, Ancient Greek Scholarship. A Guide to Finding, Reading, and Understanding Scholia, Commentaries, Lexica, and Grammatical Treatises, from Their Beginnings to the Byzantine Period, Oxford-New York, Oxford University Press, 2007.

Frédéric, Madeleine, La répétition. Étude littéraire et rhétorique, Tübingen, Max Niemeyer, 1985.

Guilleux, Nicole, «Allitérations et assonances associées à l'emploi des impressifs de sonorité dans la langue homérique », in A. Blanc et E. Dupraz (éd.), Procédés synchroniques de la langue poétique en grec et en latin, Bruxelles, Safran, 2007, p. 95-102.

Hackstein, Olav, «La paréchèse et les jeux de mots chez Homère », in A. Blanc et E. Dupraz (éd.), Procédés synchroniques de la langue poétique en grec et en latin, Bruxelles, Safran, 2007, p. 103114.

Irigoin, Jean, « Du jeu verbal à la recherche étymologique : Homère et les scholies homériques », Revue de Philologie 65/1, 1991, p. 129-134.

Navarre, Octave, La Rhétorique grecque avant Aristote, Paris, Hachette, 1900.

Patillon, Michel, La Théorie du discours chez Hermogène le Rhéteur: essai sur les structures linguistiques de la rhétorique ancienne, Paris, Les Belles Lettres, 1988.

Packard, David W., «Sound-patterns in Homer», Transactions and Proceedings of the American Philological Association 104, 1974, p. 239-260.

Rank, Louis Philippe, Etymologiseering en serwante Verschijnselen bij Homerus, Assen, Van Gorcum, 1951.

Saussure, Ferdinand de, Anagrammes homériques, éd. Pierre-Yves Testenoire, Limoges, LambertLucas, 2013.

Standford, William Bedell, « Greek views on Euphony », Hermathena 61, 1943, p. 3-20.

Testenoire, Pierre-Yves, « Des anagrammes chez Homère ? De Saussure aux commentateurs anciens », Lalies, 30, 2010, p. 215-231. 